\title{
Clinical Effect of Patented Product for Specialized Nursing in Operating Rooms
}

\author{
Lin Ke, Fan Qi ${ }^{*}$, Wang Fang, Ding Zhaokai, Bai Jing \\ Operating Room, The Second Affiliated Hospital of Kunming Medical University, Kunming, China
}

Email address:

77340786@qq.com (Fan Qi)

${ }^{*}$ Corresponding author

\section{To cite this article:}

Lin Ke, Fan Qi, Wang Fang, Ding Zhaokai, Bai Jing. Clinical Effect of Patented Product for Specialized Nursing in Operating Rooms. American Journal of Nursing Science. Vol. 9, No. 6, 2020, pp. 423-428. doi: 10.11648/j.ajns.20200906.16

Received: November 9, 2020; Accepted: November 24, 2020; Published: December 4, 2020

\begin{abstract}
Background: Surgical counts are a basic and crucial specialized nursing operation in operating rooms, to guarantee surgical safety, prevent surgical items from retaining in the body and ensure the success of surgery and safety of patients. Objective: To explore the effect of "Surgical Instruments Organizer" applied in operating rooms as a nursing patented product to minimize the occurrence of surgical counts missing. Methods: Research setting was arranged in a large general hospital where two of the emergency operating rooms were used to compare the effects and influences of the application with "Surgical Instruments Organizer". Results: The rate of surgical counts missing in the control room was significantly higher than that in the experimental room $(\mathrm{P}<0.05)$. In both the control room and the experimental room, the incidence of one surgical counts missing in one surgery was higher than that of two surgical counts missing in one surgery. Surgical counts missing between two surgeries mostly happened when surgical items were removed temporarily (141, 10.2\% in the control room; 55, $4.0 \%$ in the experimental room), while surgical counts missing happened the least in preoperative preparation $(21,1.5 \%$ in the control room; $4,0.3 \%$ in the experimental room). Between two operating rooms, most of the time lost items were found by scrub nurses $(403,29.2 \%$ in the control room; $165,12.1 \%$ in the experimental room). Most of lost items can be found within 10 minutes $(312,22.6 \%$ in the control room; $112,8.2 \%$ in the experimental room), but a few items cost more than 60 minutes (32, 2.3\% in the control room; 17 , $1.3 \%$ in the experimental room). Conclusion: "Surgical Instruments Organizer" can effectively reduce the rate of surgical counts missing in emergency surgeries, and improve the nursing quality and surgical safety.
\end{abstract}

Keywords: Emergency Surgery, Surgical Instruments Organizer, Surgical Counts Missing, Nursing Safety

\section{Introduction}

Surgical counts are a basic and crucial specialized nursing operation in operating rooms, to guarantee surgical safety, prevent surgical items from retaining in the body and ensure the success of surgery and safety of surgical patients [1]. Due to the rapid development of surgeries, the complexity of specialized nursing operation increases. The content and quantity of surgical counts also increase and become complicated. A lot of miscellaneous surgical items are added, on the basis of surgical dressings and surgical instruments alone previously, namely, everything that is likely to retain in surgical incisions, such as blocking belts, hanging belts, urinary catheters and syringes, etc. [2]. The increase of the quantity and species of aseptic items on operating tables brings greater and more risks and safety hazards to the safe operation of surgical counts. These risks and safety hazards are especially prominent in emergency surgeries [3]. In emergency surgeries, since patients are seriously ill and their life is endangered, they need surgeries in the shortest time. However, it is often the case that preoperative preparations are insufficient and accidents often happen, for example, surgical methods change temporarily, patients had sudden bleeding and their vital signs become unstable, making the species and quantity of surgical items change temporarily, which greatly increases the difficulty and risk of surgical counts. Surgical counts missing often happens, too.

Surgical counts missing refers to the occurrence of counts missing, e.g., items are missing, increased, inconsistent in quantity, quality and character, etc., or inconsistent with records or checks, when all kinds of surgical items (surgical 
instruments, surgical suture needles, surgical dressings, surgical scalpels and miscellaneous items during surgeries, etc.) are counted in different times of a variety of surgeries. After repeated check and lookup, the mistake is corrected before a surgical incision is sutured. It affects the normal operation of surgeries, but doesn't cause serious adverse outcomes, for example, a foreign matter remains in the body $[4,5]$. Although surgical counts missing doesn't really cause a foreign matter to remain in the body, it will trigger many serious complications, such as infections, septicemia, long length of stay, intestinal obstruction, viscera perforation and even death, due to additional surgical procedures inside and outside incisions, object movement in the aseptic area of surgery and elongation of surgery time [6, 7]. In addition, unsuccessful surgical counts will make a surgical team suspend the scheduled surgical procedures and spend a lot of extra time finding lost items, which prolongs the surgery and anesthesia time and increases adverse reactions to anesthesia [8]. Additional surgery costs may be incurred because $\mathrm{C}$-arm X-ray equipment looks for items left in surgeries and the dosage of anesthesia is increased. Therefore, surgical counts missing hinder the safe operation of surgeries from multiple aspects and cause serious consequences to surgical treatment.

To minimize the occurrence of surgical counts missing in operating room nursing, effectively reduce and control operating room nursing risks and improve the efficiency of surgical procedures, operating rooms of our hospital designed and made Surgical Instruments Organizer, a patented nursing product approved by State Intellectual Property Office (Patent No.: 201620526606.3), according to the requirements of actual surgeries and species and quantity of common surgical items. From January to June 2017, two emergency operating rooms open 24 hours in a central operating room of a large general hospital in Yunnan Province were used as "experimental room" and "control room", to test the clinical applicability and surgical safety of this patented product, with a view to explore the role and effect of this patented product for specialized nursing in operating rooms on reducing surgical risks and hazards, improve surgical safety and obtain an evidence ground for clinical promotion and application.

\section{Methods}

\subsection{Data Collection}

The data collection in the present study was done in an operating room of a large general hospital in Yunnan Province. In our hospital, the central operating room was a modern laminar flow clean operating room. There were 29 operating rooms, two of which were dedicated for emergency surgeries. The data were collected with the following procedures: (1) Researchers filed a written application to people in the nursing department and operating rooms of the surveyed hospital and secure the consent of the hospital and department; (2) Researchers submitted a written application for "experiments" to Ethics
Committee of the surveyed hospital and obtained an experimental qualification; (3) Researchers enrolled 2 assistants (by means of voluntary application) to assist them in finishing the experiment in operating rooms;(4) Researchers chose two emergency operating rooms with basically the same layouts, indoor items and facilities. These emergency operating rooms undertook emergency surgeries for 24 hours from Monday to Sunday; (5) One of the two operating rooms was selected as "“"the experimental room" and the other was "the control room"; (6) We used Surgical Instruments Organizer in "the experimental room", to place sterile tables and cooperate with surgeries, while the "the control room" still followed routine operating room nursing procedures in our hospital. (7) We designed "Emergency Surgeries Counts Missing Record Book", which recorded surgical departments and surgical types with surgical counts missing, the number of surgical counts missing, time of missing, surgical team members who found lost items, places where lost items were found and extra time to look for lost items. The record book was placed in the "the experimental room" and "the control room". The nurses who took care of emergency surgeries kept records according to intraoperative conditions. (8) We collected, tallied and recorded data in the "Emergency Surgeries Counts Missing Record Book" every week. Meanwhile, they observed and guided the use of Surgical Instruments Organizer, to ensure the effectiveness of clinical use.

\subsection{The Design and Making of Surgical Instruments Organizer}

Researchers reviewed records about surgical counts missing, relevant nursing errors and adverse events in operating rooms of a large general hospital in Yunnan Province over the last 5 years, consulted relevant literature, did statistics and analysis and summarized species and quantities of items missing in surgeries, designed and made a "Surgical Instruments Organizer": (1) The utensil was made of heat-resistant and sterile transparent plastic, cushion and metal clip, etc. (2) The Surgical Instruments Organizer was $50 \mathrm{~cm}$ long, $20 \mathrm{~cm}$ wide and $4 \mathrm{~cm}$ tall. It was a flat box. (3) The box was divided into left and right cells vertically by the width of $20 \mathrm{~cm}$. The right side was divided into upper and lower cells by the length of $40 \mathrm{~cm}$. The left cell and lower right cell had covers. (4) The left cell was made up of four long and narrow boxes in parallel. Each box was $2 \mathrm{~cm}$ wide. The lower end was not closed. The front and back ends of long and narrow boxes were lock blocks made of metal clips. Surgical sutures with four specifications can be placed in the boxes. (5) The upper right cell didn't have a cover. Syringes, needles, decollement tools and various catheters, etc. can be placed in it. The lower right cell had a cover. A cushion was placed in the box. All kinds of surgical suture needles and threaded needles were inserted in the cushion. (Figures 1-4) 


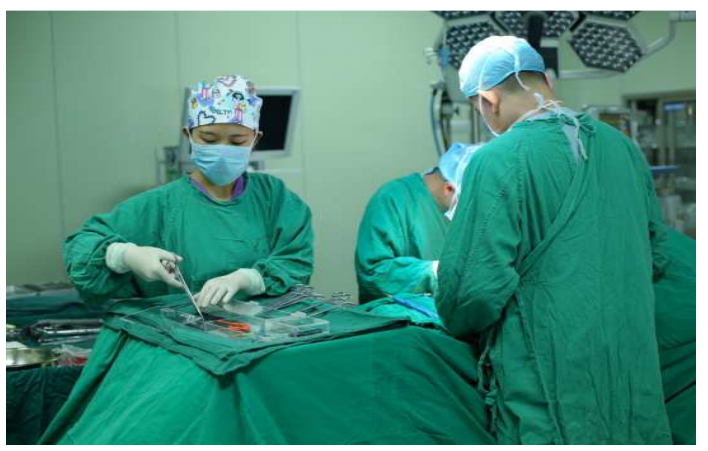

Figure 1. Surgical Instruments Organizer used in the emergency operation.

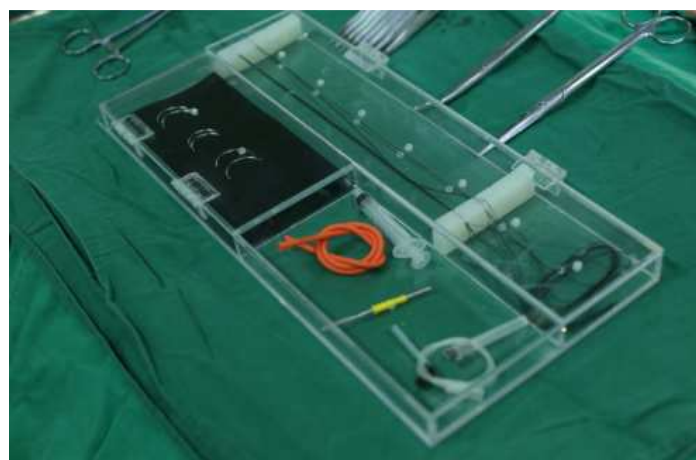

Figure 2. Full view of Surgical Instruments Organizer.

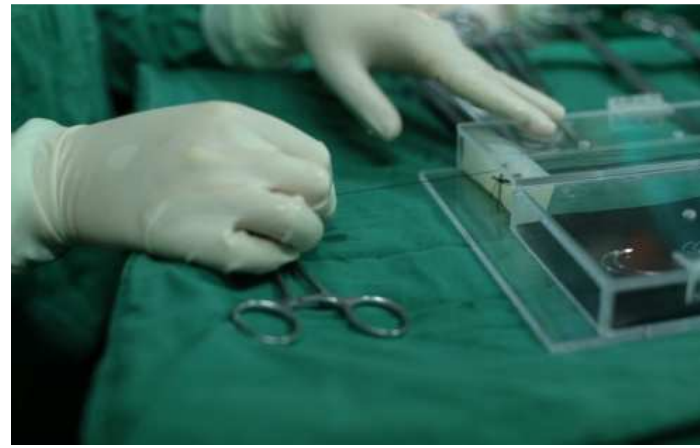

Figure 3. Sutures can be appropriately used in Surgical Instruments Organizer.

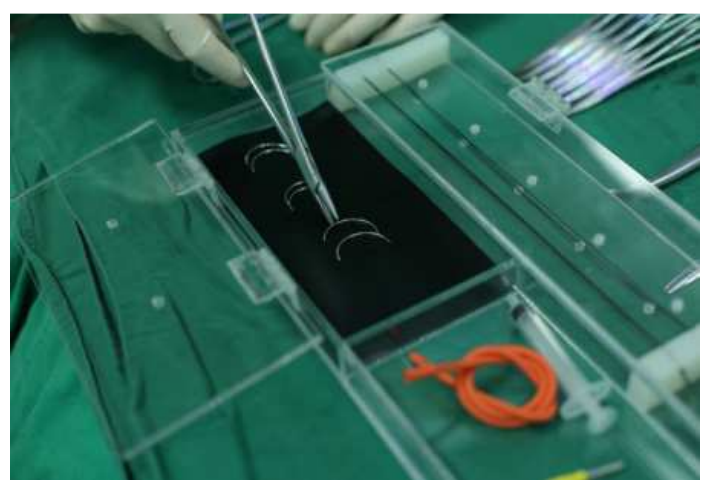

Figure 4. Surgical needle and miscellaneous surgical items can be appropriately used in Surgical Instruments Organizer.

\subsection{Statistical Analysis}

Statistical analysis was performed using the statistical software SPSS19.0. A descriptive analysis was conducted on the general data of all described objects. Enumeration data about the occurrence and results of surgical counts missing in the experimental group and control group were analyzed by chi-squared test.

\section{Results}

\subsection{Types of Emergency Surgeries}

In our study, we selected two emergency operating rooms from operating rooms of a large general hospital in Yunnan Province as the experimental room and the control room from January to June, 2017. 2744 emergency surgeries were collected as experimental data, including 1381 in the control room and 1363 in the experimental room. Among all types of emergency surgeries, the proportion of obstetric emergency surgeries was the highest, i.e., $323(23.4 \%)$ in the control room and $296(21.6 \%)$ in the experimental room. The proportion of urologic surgeries was the lowest, i.e., 150 $(10.09 \%)$ in the control room and $174(12.8 \%)$ in the experimental room (Table 1).

Table 1. Types of Emergency Surgeries $(N=2744)$.

\begin{tabular}{lllll}
\hline \multirow{2}{*}{ Surgical Departments } & Control Group & & Experimental Group & Percentage\% \\
\cline { 2 - 5 } & Frequency & Percentage\% & Frequency & 21.6 \\
\hline Obstetrics & 323 & 23.4 & 296 & 14.1 \\
Gynaecology & 172 & 12.4 & 192 & 16.1 \\
Orthopedic Surgery & 192 & 13.9 & 219 & 12.8 \\
Urology & 150 & 10.9 & 174 & 15.7 \\
General Surgery & 232 & 16.8 & 214 & 19.7 \\
Neurosurgery & 312 & 22.6 & 268 & 100 \\
Total & 1381 & 100 & 1363 & \\
\hline
\end{tabular}

\subsection{Survey Results of Surgical Counts Missing}

Our results showed that the surgical counts missing in the control room $(585,57.6 \%)$ was significantly higher than that in the experimental room $(237,17.4 \%)$ and the difference was statistically significant $(\mathrm{P}<0.05)$. In both the control room and the experimental room, the incidence of one surgical counts missing in one surgery was higher than that of two surgical counts missing in one surgery. Surgical counts missing between two surgeries mostly happened when surgical items were removed temporarily $(141,10.2 \%$ in the control room; 55, $4.0 \%$ in the experimental room), while surgical counts missing happened the least in preoperative preparation $(21,1.5 \%$ in the control room; $4,0.3 \%$ in the experimental room). Between two operating rooms, most of 
the time lost items were found by scrub nurses (403, 29.2\% in the control room; $165,12.1 \%$ in the experimental room). While surgeons and anesthesiologists were surgical team members who had the least opportunity to find lost items. Most of lost items were found on surgical instrument carriages of scrub nurses $(241,17.4 \%$ in the control room; 94 , $6.9 \%$ in the experimental room). There were few cases that lost items were found inside surgical incisions $(16,1.1 \%$ in the control room; $4,0.3 \%$ in the experimental room). Most of lost items can be found within 10 minutes $(312,22.6 \%$ in the control room; $112,8.2 \%$ in the experimental room), but a few items cost more than 60 minutes $(32,2.3 \%$ in the control room; $17,1.3 \%$ in the experimental room).

Table 2. Comparison of Variables for Surgical Counts Missing between the Control Room and Experimental Room (N=2744).

\begin{tabular}{|c|c|c|c|c|c|c|c|}
\hline \multirow{2}{*}{ Variable } & \multicolumn{2}{|c|}{ Control Group (N=1381) } & \multicolumn{5}{|c|}{ Experimental Group (N=1363) } \\
\hline & Frequency & Percentage $\%$ & Frequency & Percentage $\%$ & $\mathbf{X}^{2}$ & df & $\mathbf{P}$ \\
\hline Emergency surgeries without surgical counts missing & 796 & 57.6 & 1126 & 82.6 & 31.31 & 1 & 0.00 \\
\hline $\begin{array}{l}\text { Emergency surgeries with surgical counts missing } \\
\text { Types of surgical counts missing }\end{array}$ & 585 & 42.4 & 237 & 17.4 & & & \\
\hline Two missing in one surgery & 61 & 4.4 & 14 & 1.0 & 32.26 & 2 & 0.00 \\
\hline One missing in one surgery & 524 & 38.0 & 223 & 16.4 & & & \\
\hline \multicolumn{8}{|l|}{ Time of surgical counts missing } \\
\hline When counting before a scalpel was used & 81 & 5.8 & 22 & 1.6 & & & \\
\hline When adding items in a surgery & 141 & 10.2 & 55 & 4.0 & & & \\
\hline When removing items in a surgery & 132 & 9.6 & 49 & 3.6 & & & \\
\hline Before an incision/coelom was closed & 101 & 7.3 & 45 & 3.3 & & & \\
\hline After an incision/coelom was closed & 60 & 4.4 & 53 & 3.9 & & & \\
\hline Before suturing skin & 49 & 3.6 & 9 & 0.7 & & & \\
\hline \multicolumn{8}{|l|}{ Surgical team members who found lost items } \\
\hline Surgeon & 30 & 2.2 & 4 & 0.3 & & & \\
\hline Surgical assistant & 40 & 0002.9 & 18 & 1.3 & & & \\
\hline Scrub nurse & 403 & 29.2 & 165 & 12.1 & & & \\
\hline Circulating nurse & 91 & 6.6 & 35 & 2.6 & & & \\
\hline \multicolumn{8}{|l|}{ Places where surgical items were lost } \\
\hline Inside an incision & 16 & 1.1 & 4 & 0.3 & 33.86 & 5 & 0.00 \\
\hline Inside a sterile area around the incision & 140 & 10.2 & 41 & 3.0 & & & \\
\hline Inside a sterile area on a surgical instrument tray & 140 & 10.2 & 76 & 5.6 & & & \\
\hline Inside a sterile area on a surgical instrument carriage & 241 & 17.4 & 94 & 6.9 & & & \\
\hline Outside the sterile area & 48 & 3.5 & 22 & 1.6 & & & \\
\hline \multicolumn{8}{|l|}{ Time used to find lost items } \\
\hline $1-10$ mins & 312 & 22.6 & 112 & 8.2 & 32.07 & 4 & 0.00 \\
\hline $11-30 \mathrm{mins}$ & 161 & 11.7 & 67 & 4.9 & & & \\
\hline $31-60 \mathrm{mins}$ & 80 & 5.8 & 41 & 3.0 & & & \\
\hline$>60$ mins & 32 & 2.3 & 17 & 1.3 & & & \\
\hline
\end{tabular}

\section{Discussion}

\subsection{The Clinical Application of Surgical Instruments Organizer}

Our study showed that the total surgical counts missing in the control room was significantly higher than that in the experimental room and the difference was statistically significant $(\mathrm{P} \leq 0.05)$. This result proved that Surgical Instruments Organizer played an effective role in reducing the occurrence of surgical counts missing. Surgical Instruments Organizer was made of transparent materials, so that scrub nurses and circulating nurses can see the use and quantity of contents at a glance during surgeries. Even in an emergency, the number of some small items which were easily lost can be observed at any time. The use of these surgical items can be observed dynamically. Meanwhile, surgical counts missing can be effectively avoided.

The cell design of Surgical Instruments Organizer also allowed suture needles, threaded needles, syringe needles, "peanut-like" decollement tools and other small miscellaneous items used in a surgery to be placed in a specialized place. During surgeries, these items wouldn't be chaotic or lost, due to the small size and complicated types. Besides, a storage groove especially designed for sutures can fix different types of sutures to the groove. In doing this, nurses can fetch and replace different types of sutures, prevent sutures from intertwining due to the replacement and addition of sutures in an emergency, which may affect the effectiveness of sutures and increase the cleanliness of items on a sterile tray. In a word, using Surgical Instruments Organizer clinically can avoid the loss of surgical items and discrepancy in surgical counts in emergency surgeries from different angles in different ways, thereby reducing the occurrence of surgical counts missing and ensuring the safety of surgical procedures.

\subsection{An Analysis of the Results of Surgical Counts Missing}

Our results showed that among all surveyed surgical departments, the occurrences of emergency surgeries and 
surgical counts missing in obstetrics were higher than those of other surgical types. This phenomenon was mainly because the number of obstetrical surgeries had surged since China published the two-child policy [9]. Many elderly pregnant women with obstetric risks joined the rank of second pregnance and lowered the rate of eutocia. The number of cesarean sections increased. Meanwhile, the incidence of serious complications from secondary pregnancy rose, such as re-pregnancy of scarred uterus, placenta previa and placental implantation, and the risk of cesarean section increased [10]. In operating room nursing, to cope with unexpected surgical accidents, such as hysterorrhexis and intraoperative hemorrhage, etc., intraoperative nursing and difficulty in cooperating with surgical technique increased, leading to high incidence of surgical counts missing.

In terms of the incidence of surgical counts missing, neurosurgical surgeries were second only to obstetric surgeries. Emergency surgeries of neurosurgery mainly included craniocerebral trauma, spontaneous rupture and bleeding of intracranial vessels and intracranial oncothlipsis, etc. Most patients were critically ill. Apart from different degrees of dysneuria, they were complicated with severe physiological dysfunctions, such as respiration, circulation, metabolism and endocrine. The intraoperative conditions were critical and the rescue time was pressing [11]. During emergency surgeries, operating room nurses must closely cooperate with surgical procedures, prepare and add first aid materials and extra surgical instruments, items, suture needles and hemostatic items, etc., when patients had surgical accidents and unexpected surgical risks. In this case, operating room nurses may encounter surgical counts missing due to a lack of sufficient professional experience and adept operational skills. Or the medical staff were anxious to cope with the rescue work before an effective counting time was arranged, which led to surgical counts missing. For surgical counts missing between two surgeries, the proportion of one surgical counts missing in one surgery was higher than that of two surgical counts missing in one surgery, which suggested that two surgical counts missing in one surgery was clinically rare in the surveyed general hospital of our study. If a surgical counts missing had already happened in the surgery, it would certainly bring unnecessary obstacles to the whole surgical team. The medical staff must spend extra time and effort searching for lost items, which not only increased the burden of the whole team, but also posed a risk of infection control [12]. Therefore, in subsequent surgical procedures, the whole surgical team shall do their best to avoid same mistakes.

In the present study, we investigated different times of surgical counts missing and found that the increase or decrease of surgical items in the midway of surgery was an important time node that increased the frequency of surgical counts missing. This finding was very similar to the finding about surgical counts missing reported previously [4]. It was reported that the score of surgical counts missing would rise, when different types of surgical items were added unexpectedly or removed from an operating table during a surgery. Generally speaking, items on an operating table must be prepared before a surgery and counted by the scrub nurse and circulating nurse jointly, during preoperative preparation and before a surgeon used the scalpel [2]. During preoperative counting, the scrub nurse and circulating nurse often had sufficient time and a good and conscious mental state to count surgical items. After both parties checked and counted, they kept a detailed written record of surgical items, so the incidence of surgical counts missing was low. The increase or decrease of surgical items during a surgery was not merely a temporary change of the species and quantity of surgical items unexpectedly. In this case, the scrub nurse and circulating nurse were occupied with coordinating with surgeries and rescue work. They didn't have sufficient time and energy to check these temporarily added items and thus resulted in surgical counts missing. This situation was especially prominent in emergency surgeries, because the expected procedures and actual operation of emergency surgeries were quite different. During a surgery, patient's conditions were not stable. The incidence of unexpected conditions was high. Operating room nurses need spend more time and energy disposing accidents during emergency surgeries and focus on emergency operations. They would ignore surgical items, leading to surgical counts missing [13].

The research finding also suggested that surgical items were most often lost in surgical instrument carriages and surgical instrument trays. Surgical items were seldom lost around or inside surgical incisions. This finding was mainly due to the frequency and time surgical items were placed in different locations. Surgical instrument carriages were used to place intraoperative items, while surgical items on surgical trays were items required in the current surgical procedure [14]. Both of them were places where surgical items were frequently placed. On the other hand, surgical items were seldom placed around or inside a surgical incision for a long time. Especially with the rapid development of modern minimally invasive surgeries, in many endoscopic surgeries, extra items wouldn't be placed around or inside a surgical incision for a long time.

When analyzing surgical team members, it was found that scrub nurses were surgical team members who most often found lost items, while anesthesiologists were members who least often found lost items. This result was directly related to different job duties of surgical team members. Scrub nurses were those who directly operated and managed surgical items. All surgical items were not delivered to surgeons until they were counted and sorted by scrub nurses [2].

Scrub nurses were most familiar with the quantity, species and use of surgical items. Also they took on the main responsibility for surgical counts. Therefore, when items were lost, scrub nurses were most aware of the reason and direction of loss. Although circulating nurses also checked items in every counting and bore the same duty for counting, they didn't actually operate or manage items, so they were not as familiar with the use of items as scrub nurses. Although in a surgical team, it was surgeons who actually 
used surgical items, they paid more attention to surgical procedures, instead of the use and status of items. Likewise, the duty of anesthetists was to ensure the intraoperative anesthetic effect and safety of patients. They would pay even less attention to the use of surgical items than surgeons. Therefore, when surgical items were lost, scrub nurses, with the closest relationship with surgical counts, were also surgical team members who first found lost items.

\section{Conclusion}

"Surgical Instruments Organizer" is a specialized nursing utensil that can effectively reduce the rate of surgical counts missing in emergency surgeries. The use of "Surgical Instruments Organizer" in emergency surgeries and high-risk complex surgeries will undoubtedly help operating room nurses cooperate with surgeries safely and effectively, lower surgical risks and improve the quality of specialized nursing. Therefore, this patented product for specialized nursing in operating rooms is worthy of clinical popularization, so that it can truly contribute to clinical practice, improve nursing safety, promote patients' recovery and bring more and better economic benefits and social benefits.

Drawing on the several "risk times" of surgical counts missing detected in our study, we suggest that operating room nurses should pay high attention to "risk times" in clinical nursing, improve risk awareness, avoid surgical counts missing and lower surgical risks. In addition, we can also set up a corresponding lookup process, according to places where surgical items are frequently lost, find lost items targetedly in a proper order and improve the finding rate of lost items. Meanwhile, operating room nursing managers may also use our research findings as an evidence ground, to improve and perfect the safety management specifications and procedures for clinical nursing in operating rooms, improving the quality of nursing management and refine safety content in specialized departments.

\section{References}

[1] Freitas PS, Silveira RC, Clark AM, Galvão CM. Surgical count process for prevention of retained surgical items: an integrative review. Journal of Clinical Nursing, 2016, 25 (1314): $1835-1847$.
[2] Specialized Committee for Operating Rooms, Chinese Nurse Association, Guide to Operating Room Nursing Practice, 2016 Edition. Beijing: People's Medical Publishing House, 2018, 108-109.

[3] Li, Wenhong, Li Lina, Huang Meijuan, Liu Yuanyuan and Xu Yanru, The application of risk management in emergency surgeries. Chinese Journal of General Practice, 2011, 9 (1): 133-134.

[4] Victoria MS \& Joseph JC. Designing a Safer Process to Prevent Retained Surgical Sponges: A Healthcare Failure Mode and Effect Analysis. AORN J, 2011, 94: 132-141.

[5] AORN. Recommended practices for prevention of retained surgical items. In: Perioperative Standards and Recommended Practices. Denver, CO: AORN, Inc, 2015: 263-282.

[6] Lincourt AE, Harrell A, Cristiano J, Sechrist C, Kercher K, Heniford BT. Retained foreign bodies after surgery. J Surg Res. 2007, 138 (2): 170-174.

[7] Gawande AA, Studdert DM, Orav EJ, Brennan TA, Zinner MJ. Risk factors for retained instruments and sponges after surgery. N Engl J Med. 2003; 348 (3): 229-235.

[8] Victoria MS, Ann GS, Yelena P, Hillary ES, Michelle M. The hidden costs of reconciling surgical sponge counts. AONR J, 2015, 102 (5): 498-506.

[9] Meng, Qian and Lin Peng, A survey of different distributions of high-risk pregnant women with and without two-child policy. Maternal \& Child Health Care of China, 2016, 31 (20): 4266-4268.

[10] Wang, Lijing, The risk of re-pregnancy of scarred uterus and its influence on pregnancy outcomes. Beijing Medical Journal, 2016, 38 (1): 92-94.

[11] Luo, Lizhen and Yan Xiaoyu. Nursing problems in emergency surgeries of neurosurgery and countermeasures. The $10^{\text {th }}$ National Academic Exchange and Symposium for Operating Room Nursing 2006.

[12] Pan, Xiaoling, Yang Liqin and Liu Ping, The application of failure mode and effect analysis during surgical counts, Journal of Qilu Nursing, 2014, 20 (14): 118-119.

[13] $\mathrm{Wu}$, Meichan and Lin Yongqin. Training on the operating room nurses' ability to cope with emergency surgeries. Journal of Qilu Nursing, 2014, 20 (14): 120-121.

[14] Specialized Committee for Operating Rooms, Chinese Nurse Association, Guide to Operating Room Nursing Practice, 2017 Edition. Beijing: People's Medical Publishing House, 2017, 20-22. 